\title{
Examining the productiveness of student resources in a problem-solving interview
}

\author{
Lisa M. Goodhew, ${ }^{1}$ Amy D. Robertson, ${ }^{2}$ Paula R.L. Heron, ${ }^{1}$ and Rachel E. Scherr ${ }^{2}$
}

${ }^{1}$ Department of Physics, University of Washington, 3910 15th Ave NE, Seattle, WA, 98192

${ }^{2}$ Department of Physics, Seattle Pacific University, 3307 3rd Ave W, Seattle, WA, 98119

\begin{abstract}
It is central to the resources theoretical framework that the knowledge elements that comprise thinking-i.e., resources - are sensible, based on experience, and continuous with formal physics. While many authors agree that students' conceptual resources can be framed as continuous with formal physics, few have discussed how and in what ways specific resources are productive for learning. We closely examine the progression of one introductory physics student's thinking during an exploratory problem-solving interview, attending to the role that specific conceptual resources play in the evolution of her ideas over short time-scales. Two commonly activated resources for wave propagation (treating a pulse as an object moving through a medium and treating a pulse as a propagating disturbance) are integral to the conceptual progress she makes during this episode, and therefore can be considered productive for this situation.
\end{abstract}

\section{INTRODUCTION}

While the literature that develops the resources theory of knowledge is multi-faceted, and thus does not offer a single synoptic definition of a resource, there are several points of consensus regarding what resources are. Resources are described as knowledge elements or ideas at a smaller grain size than scientific concepts, which are activated in the moment to construct explanations, arguments, and concepts [1-3]. Activation of a particular resource is context-sensitive, influenced by the learner themselves, the environment, and the question at hand [4]. Across the literature, resources are depicted as neither inherently correct nor incorrect; instead, they may be appropriately or inappropriately activated for a particular question. Though resources are neither correct nor incorrect, they are described as inherently sensible (reasonable to the learner) and continuous with formal physics ideas, such that there exists a trajectory over which a "raw" resource can become a scientific concept $[1,2,5]$.

In previous work we identified two common conceptual resources for wave propagation from student responses to the "pulse-flick question" (Fig. 1), a conceptual question about wave propagation used in previous studies [6]. These resources are: (A) treating a pulse as a macroscopic object moving in medium whose properties affect the pulse's speed (object-in-medium), and (B) treating a pulse as a propagating local disturbance in a medium (propagating-disturbance) [7]. Each of these resources is continuous with formal physics concepts about mechanical wave propagation: object-in-medium may represent the beginnings of understanding that wave speed is determined by properties of the medium. Propagating-disturbance appropriately describes the nature of pulses and may represent the beginnings of a canonical model for propagation.

Some authors who report particular conceptual resources describe how they are continuous with canonical physics or might be productive for students, but authors have less commonly illustrated how these resources are productive in context. This paper presents an illustrative case of the productiveness of the resources object-in-medium and propagating-disturbance, and thereby demonstrates how theoretical notions of productiveness may be realized in context. We analyze a short (approximately 20-minute) excerpt from an exploratory problem-solving interview with one introductory physics student, pseudonymed Sophie. We identify instantiations of these two conceptual resources and give evidence that these resources are both (i) appropriately activated for the question at hand and (ii) contribute to the development of Sophie's thinking. We claim that because these two resources are appropriately activated and contribute to Sophie's thinking, they are productive for her.

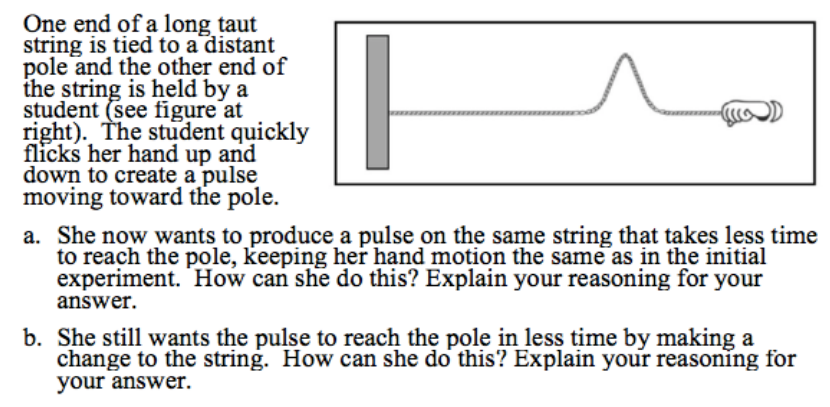

FIG 1. The pulse-flick question, modified from [6].

\section{PRODUCTIVENESS OF RESOURCES}

While resources are thought of as inherently continuous with formal physics, assessing their productiveness - the extent to which a resource is appropriately activated or plays a role in progress - requires examining the context in which they are activated. To do this, we draw on the work of Harrer [8], who defines two types of productiveness: disciplinary productiveness and situated productiveness.

\section{A. Disciplinary Productiveness}

Harrer defines disciplinary productiveness as "appropriate activation [of a resource] in a particular context, as judged by the community of physicists via the instructor or researcher" [8]. Thus, disciplinary productiveness is not an attribute of the resource itself, but of the resource-in-context. 
For example, the resource closer means stronger [1] is productive for explaining why the air is warmer closer to a fire, but not for explaining why it is warmer on Earth during the summer. While disciplinary productiveness is evaluated in light of a resource's consistency with relevant canonical concepts, we do not interpret this to mean that the student must be "almost there" [8,9] for a resource to be considered productive in this sense. In this paper, we use disciplinary productiveness to assess the appropriateness of these two resources for reasoning about pulse propagation.

\section{B. Situated Productiveness}

Harrer defines situated productiveness of resources as a contribution to intellectual progress toward an (often emergent) goal that is implicitly or explicitly defined by students themselves [8]. Situated productiveness is discussed in Harrer's work in the context of group interactions: "If a resource allows the group to make significant progress toward an emergent, shared goal...it can be considered productive for the situation at hand" [8]. In other words, situated productiveness is evaluated in light of the role a resource plays in a student's or group's evolving thinking. Thus, it is appropriate to use this construct in situations where students' unfolding thinking is on display, whether this occurs in group interactions or in instances where students verbalize their thought processes individually. In this paper, we use the construct of situated productiveness to assess two conceptual resources' contribution to a student's progress toward answering an emergent question.

\section{MEHODOLOGY \& EXCERPT SELECTION}

In this paper, we use interview excerpts to (1) demonstrate disciplinary and situated productiveness in the context of mechanical wave propagation and in doing so (2) provide proof-of-concept of the productiveness of two resources for understanding mechanical waves. Thus, the objective of this work is to illustrate theoretical notions of productiveness by examining a case of productiveness of two particular conceptual resources. The transcript we analyze for this case-study comes from one of five exploratory problem-solving interviews conducted with students enrolled in a third quarter, calculus-based introductory physics course at the University of Washington. Interviews were unrelated to the coursework or grades, and student volunteers were selected on a first-come basis. The interview with Sophie took place after she had received some instruction on wave mechanics. In the interview, Sophie was given two conceptual questions about mechanical waves and asked to "think aloud" about her answers. The interview was video-recorded and lasted about 40 minutes. The excerpts presented here come from Sophie's response to the second of two questions she was given in her interview (Shown in Fig. 1). We chose these particular excerpts because in them Sophie articulates (and then resolves) an emergent question, and she uses the two common resources we have reported.
Since Sophie uses these two resources in the context of wave propagation, these excerpts have the potential to demonstrate the disciplinary productiveness of the resources. Since Sophie uses these two resources to articulate and resolve an emergent question, these excerpts have the potential to demonstrate the situated productiveness of these resources.

To analyze the disciplinary productiveness of the two resources, we examine their specific instantiations against a disciplinary understanding of pulse propagation: the pulse can be modeled as transverse displacement of sequential parts of the medium governed by the laws of mechanics, such that pulse speed is determined by properties of the medium.

To analyze the situated productiveness of these resources, we examine the ways in which they contribute to the progression of Sophie's thinking during her interview. We draw on Odden and Russ's notion of a "vexation point" to understand a tension Sophie articulates as the beginning of a process of sense-making and knowledge construction. The vexation point is defined as the "critical moment...when [she] attend[s] to and articulate[s] an inconsistency or gap in [her] understanding" which signals a shift from recalling previously learned knowledge to constructing new knowledge [10]. Therefore, in our analysis we attend to the role these resources play in Sophie's knowledge-building following a vexation point in her interview.

\section{IV.ANALYSIS}

In the following excerpts Sophie (denoted by $\mathrm{S}$ in the transcript) discusses the pulse-flick question (Figure 1) with author LMG. After reading the question, Sophie says:

$\mathrm{S}$ : Instinctually we wanna say, flick it harder, um, because you think that you can affect the speed by adding more force into something, but you already kind of know that speed is, only depends on the medium. So trying to make the pulse faster wouldn't really do anything.

Sophie's idea that flicking harder will make the pulse move faster can be interpreted as treating the pulse as a Newtonian object, which moves faster when thrown with greater force [11]. She articulates a dissonance between her intuitive thinking and her learned response that only the medium affects pulse speed. This is the vexation point: she articulates something that does not "make sense," and (in what follows) begins to sense-make about why tension should affect pulse speed but the motion of the source of the pulse should not. After some discussion, Sophie sighs and says, "The answer is increase the tension." Then she starts to explain her thinking about tension:

S: Oh! I can answer this! With music...I really like instruments. Um, in order to make the pitch for a string higher, you need to tune it by tightening it. So you're adding tension by tightening the string. The frequency...it's related to the speed. So, if you wanted to make it go faster, going at a higher frequency, you've gotta make the string tighter.

Sophie expands on her ideas about instruments, but seems dissatisfied because she says, 
S: Now to try and explain that without any, without music, because music seems to be a really easy answer, how that works, um...okay! Let's use roads.

Sophie continues, saying:

S: Yeah. Um, an un-tense road is kind of wavy. You're gonna slow down at the turns. If you add tension, the road becomes straighter and you can just zoom through it... without having to decelerate at any of the turns.

In this statement, Sophie treats the slack as a property or part of the medium that impedes the pulse moving through it, using the resource object-in-medium. While a pulse is ontologically unlike a car on a road (i.e., pulses are not Newtonian objects), Sophie uses this analogy to associate the tension of the string with the speed of the pulse in a causal manner. For this reason, we consider this resource appropriately activated and disciplinarily productive. The resource object-in-medium also helps Sophie to connect her initial ideas, which treat pulses like objects, to ideas about the effect of the medium on pulse speed. In what follows, Sophie uses a second resource, propagating-disturbance, to expand on this idea, suggesting that her use of the second resource was in fact stimulated by the first. The interview continues with the following exchange:

LMG: So you're thinking then, tell me if this sounds like what you're thinking...if I make a tighter string, then the pulse... has less...there's less points that make it slow down.

S: Yeah. Kind of. Um, there's, there's, if we look at everything really, really, small, like we zoom in, uh-

LMG: On the string?

$\mathrm{S}:$ On the string. A string that is not completely tense will have points where it's just kind of floating there, and it will take more energy to move those than something that's kind of ready to move.

Here, Sophie begins to attend to the movement of "points" on the string - an instantiation of the resource propagating-disturbance. This resource helps Sophie elaborate on her ideas about how tension affects the speed of the pulse: tension tells her something about how "ready" the points in the medium are to move. Consideration of the dynamics of component parts of the medium is an important part of a canonical model for pulse propagation, so from a disciplinary perspective, this resource is productive here. Following this statement, Sophie continues:

LMG: Okay. So it takes more -

$\mathrm{S}$ : It's kind of like when you're doing "The Wave," and you've got people not paying attention.

LMG: Okay, so there's less, like, there's less of whatever thing it is that makes each point of the string go up and down.

S: Yeah. So it just proceeds a bit slower.

LMG: Okay!

S: It's like, if the string were a bunch of humans, because we're doing a Wave in a stadium, um, if none of the people are ready to do the Wave, they're sort of relaxed, there's a time it takes to communicate the idea that, 'Hey guys we're doing a Wave now,' so it kind of goes slowly. But if everyone's like, 'Yeah! We're gonna do the Wave! Guys, guys, Wave, woo!'

Here, Sophie builds on her thinking about the motion of particles in the medium by constructing an analogy between people participating in "The Wave" in a stadium (where successive groups of spectators briefly stand, yell, and raise their arms) and propagation of a pulse on a string. Using this analogy, Sophie implies that tension determines how quickly a disturbance of the string propagates, just as visual or verbal communication between people determines how quickly "The Wave" propagates. Although this analogy is limited"The Wave" does not propagate through physical interaction but through the choice of audience members to participateit instantiates the resource propagating-disturbance in a way that is disciplinarily productive because it supports Sophie in thinking about a pulse as sequential, transverse disturbances of parts of a medium [11]. Next, Sophie extends her analogy:

S: I guess, another example...if you're doing a Wave while holding hands with a bunch of people, um, you don't know they're pulling on your hand until they're actually doing their side of the Wave, and then you realize, 'Oh wait!' Unless you guys are all stretched out and you can clearly feel every time a person moves just a bit, because you've got nothing else, nowhere else to go, but move with them.

In this statement Sophie continues to use the resource propagating-disturbance, extending her analogy between a pulse on a string and "The Wave" in a stadium. Her extension introduces a physical interaction between discrete parts of the medium, more accurately modeling the forces between particles of the medium. This accuracy indicates the continued disciplinary productiveness of this resource.

At this point in the interview, Sophie has articulated a model for pulse propagation: discrete parts of the medium are disturbed perpendicularly to the direction of motion of the pulse, and that disturbance is communicated by physical interactions between those discrete parts. After this excerpt, Sophie reconciles her initial intuition about flicking the string harder with her answer that the tension affects the speed. She says,

S: I guess, okay, instinctively, because for some reason our brain already knows what to do, by adding more force we're actually increasing the tension in the string because we're pulling it back some more before we go. So kind of like how when you crack a whip, you don't just go like that (pulls her hand back and makes and up-and-down flicking motion). You pull it back before you go...except here it's attached, so when you pull it back, you're increasing the tension and then that makes it actually go faster...okay. I have an answer.

This statement suggests that Sophie has resolved her initial question. Whereas initially she expressed dissonance between her intuition that flicking the string harder generates a faster pulse and her recollection that only changing the medium changes the speed, here she explains how her intuition could be consistent with canonical physics. Although Sophie's thinking is not entirely complete or true in all cases ("flicking harder" does not necessarily mean 
increasing tension) it is consistent with both her initial intuition and with the equation $v=\sqrt{T / \mu}$.

Looking at the course of Sophie's thinking, we see evidence of the situated productiveness of both resources she uses: both contribute to her conceptual progress. Object-inmedium supports Sophie in connecting her ideas about the pulse as an object to ideas about the effect of the medium, which then stimulates her use of propagating-disturbance. Specifically, her ideas about the motion of small pieces of the string come from her analogy between a winding road and a slack string. Ideas about motion of parts of the medium then lead to Sophie's use of propagating-disturbance in her "Wave" analogy. Sophie uses this second resource to construct a model of a pulse as a transverse displacement of sequential parts of the medium propagated via physical interactions. Ultimately, this progression of ideas allows Sophie to answer why tension affects propagation speed, resolving the dissonance she expressed at the vexation point.

\section{DISCUSSION}

In these excerpts, we see Sophie activating two resources for mechanical wave propagation that we have reported elsewhere. Our analysis illustrates both (1) the notions of disciplinary and situated productiveness that have been described in the literature [8] and (2) the productiveness of these two resources specifically. We suspect that Sophie's interview is unique in the specific way her thinking unfolds as she discusses the pulse-flick question with the interviewer. Thus this case is not necessarily representative of a common pattern of reasoning for introductory physics students. This does not limit the theoretical significance of Sophie's interview as a case of the productiveness of these resources; Sophie's case offers an existence proof that these resources - including the canonically incorrect notion of pulses as objects - can support conceptual and situated progress. Further, we do not view Sophie's thinking as idiosyncratic: many students from several introductory physics courses use the same two conceptual resources to correctly answer several versions of the pulse-flick question in written form [7].
The analysis of interview excerpts, where we can follow a student's unfolding thinking, calls particular attention to the situated productiveness of conceptual resources. Many authors have described resources in general as productive when appropriately activated for certain physics concepts or questions (e.g., [1, 3]), and some have illustrated how specific resources are disciplinarily productive for students in context $[1,8,9,11]$. Our illustrations of disciplinary productiveness add to this literature by giving specific examples in the context of wave propagation. However, fewer authors have illustrated situated productiveness. Harrer gives some examples of situated productiveness in the context of group work. We build upon this by illustrating how two specific conceptual resources are situatedly productive for a single student in the context of a one-on-one interview.

Our case study showcases the construct of situated productiveness as a lens on the productiveness of resources that extends beyond disciplinary appropriateness. In this case, resources that are activated inappropriately can still move students' thinking forward, and thus are productive despite being incorrect. For example, some have argued that propagating-disturbance should be elicited in instruction to circumvent difficulties that may arise when students treat pulses as Newtonian objects [11]. In contrast, our analysis demonstrates that both object-in-medium and propagatingdisturbance are (situatedly) productive for Sophie; both contribute to her development of an appropriate model for pulse propagation. Thinking in terms of both disciplinary and situated productiveness may enable researchers and instructors to see student thinking as productive (or potentially so) in a broader sense.

\section{ACKNOWLEDGEMENTS}

This material is based upon work supported by the National Science Foundation Graduate Research Fellowship Program under Grant No. 1256082, and by grant DUE1608510. We wish to thank B. Harrer, T. Wan, D. Bretland, and $\mathrm{O}$. Wentink for their constructive feedback on our analysis and early drafts of this manuscript.
[1] D. Hammer, Am. J. Phys. 68, S52 (2000).

[2] A. diSessa, Cognition Instruct. 10, 105 (1993).

[3] D. Hammer et al., in Transfer of Learning from a Modern Multidisciplinary Perspective ed. J.P. Mestre (Information Age Publishing, Greenwich, 2005), pp. 89-119

[4] E. F. Redish, in Proceedings of the Enrico Fermi Summer School, Course CLVI, ed. E. Redish and M. Vicentini (Italian Physical Society, Bologna, 2004).

[5] J.P. Smith, A. diSessa, and J. Roschelle, J. Learn. Sci., 3, 2 (1993).

[6] A. Tongchai, et al., Phys. Rev. ST PER 7, 1 (2011).
[7] L. M. Goodhew, et al., in 2017 PERC Proceedings [Cincinnati, OH, July 26-27, 2017], ed. L. Ding, A. Traxler, and Y. Cao

[8] B. W. Harrer, PhD thesis, University of Maine, 2013.

[9] D. Hammer, F. Goldberg, and S. Fargason, Rev. Sci. Math. ICT Educ. 6, 51 (2012).

[10] T. O. B. Odden and R. S. Russ, in 2017 PERC Proceedings [Cincinnati, OH, July 26-27, 2017], ed L. Ding, A. Traxler, and Y. Cao.

[11] M. C. Wittmann, R. N. Steinberg, and E. F. Redish, Int. J. Sci. Educ. 25, 23 (2003). 